\title{
A CXCR4-targeted nanocarrier achieves highly selective tumor uptake in diffuse large B-cell lymphoma mouse models
}

\author{
Aïda Falgàs, ${ }^{1,2,{ }^{*}}$ Victor Pallarès,,$^{1,3, *}$ Ugutz Unzueta, ${ }^{1,2}$ \\ María Virtudes Céspedes, ${ }^{1,2}$ Irene Arroyo-Solera, ${ }^{1,2}$ María José Moreno, ${ }^{1}$ \\ Alberto Gallardo, ${ }^{1,4}$ María Antonia Mangues, ${ }^{5}$ Jorge Sierra, ${ }^{3,6}$ \\ Antonio Villaverde, ${ }^{2,7,8}$ Esther Vázquez, ${ }^{2,7,8^{*}}$ Ramon Mangues ${ }^{1,2,6, *}$ \\ and Isolda Casanova ${ }^{1,2,6}$
}

\begin{abstract}
${ }^{1}$ Biomedical Research Institute Sant Pau (IIB-Sant Pau), Hospital de la Santa Creu i Sant Pau; ${ }^{2} \mathrm{CIBER}$ en Bioingeniería, Biomateriales y Nanomedicina (CIBER-BBN); ${ }^{3}$ Department of Hematology, Hospital de la Santa Creu i Sant Pau; ${ }^{4}$ Department of Pathology, Hospital de la Santa Creu i Sant Pau; ${ }^{5}$ Department of Pharmacy, Hospital de la Santa Creu i Sant Pau; ${ }^{6}$ Josep Carreras Research Institute; ${ }^{7}$ Institut de Biotecnologia i de Biomedicina, Universitat Autònoma de Barcelona and ${ }^{8}$ Departament de Genètica i de
\end{abstract} Microbiologia, Universitat Autònoma de Barcelona, Barcelona, Spain

*AF and $\mathrm{VP}$ contributed equally to this work.

\section{Ferrata Storti Foundation}

Haematologica 2018

Volume 105(3):741-753

\section{ABSTRACT}

$\mathrm{O}$ ne-third of diffuse large B-cell lymphoma patients are refractory to initial treatment or relapse after rituximab plus cyclophosphamide, doxorubicin, vincristine and prednisone chemotherapy. In these patients, CXCR4 overexpression (CXCR4 $4^{+}$) associates with lower overall and disease-free survival. Nanomedicine pursues active targeting to selectively deliver antitumor agents to cancer cells; a novel approach that promises to revolutionize therapy by dramatically increasing drug concentration in target tumor cells. In this study, we intravenously administered a liganded protein nanocarrier (T22-GFP-H6) targeting CXCR4+ lymphoma cells in mouse models to assess its selectivity as a nanocarrier by measuring its tissue biodistribution in cancer and normal cells. No previous protein-based nanocarrier has been described as specifically targeting lymphoma cells. T22-GFP-H6 achieved a highly selective tumor uptake in a CXCR4+ lymphoma subcutaneous model, as detected by fluorescent emission. We demonstrated that tumor uptake was CXCR4-dependent because pretreatment with AMD3100, a CXCR4 antagonist, significantly reduced tumor uptake. Moreover, in contrast to CXCR $4^{+}$subcutaneous models, CXCR4 ${ }^{-}$ tumors did not accumulate the nanocarrier. Most importantly, after intravenous injection in a disseminated model, the nanocarrier accumulated and internalized in all clinically relevant organs affected by lymphoma cells with negligible distribution to unaffected tissues. Finally, we obtained antitumor effect without toxicity in a CXCR $4^{+}$lymphoma model by administration of T22-DITOX-H6, a nanoparticle incorporating a toxin with the same structure as the nanocarrier. Hence, the use of the T22-GFP-H6 nanocarrier could be a good strategy to load and deliver drugs or toxins to treat specifically CXCR4-mediated refractory or relapsed diffuse large B-cell lymphoma without systemic toxicity.

\section{Introduction}

Diffuse large B-cell lymphoma (DLBCL) represents $30-33 \%$ of all non-Hodgkin lymphomas (NHL). ${ }^{1}$ Management of DLBCL has been improved by the addition of rituximab to $\mathrm{CHOP}$ (cyclophosphamide, doxorubicin, vincristine and prednisone) chemotherapy. However, despite this advancement, R-CHOP treatment is still associated with high toxicity, relapse and an unacceptably high treatment failure

\section{Correspondence:}

RAMON MANGUES

mangues@santpau.cat

ESTHER VÁZQUEZZ

esther.vazquez@uab.cat

Received: November 19, 2018.

Accepted: June 26, 2019.

Pre-published: June 272019.

doi:10.3324/haematol.2018.211490

Check the online version for the most updated information on this article, online supplements, and information on authorship \& disclosures: www.haematologica.org/content/105/3/741

\section{(C)2020 Ferrata Storti Foundation}

Material published in Haematologica is covered by copyright. All rights are reserved to the Ferrata Storti Foundation. Use of published material is allowed under the following terms and conditions:

https://creativecommons.org/licenses/by-nc/4.0/legalcode. Copies of published material are allowed for personal or internal use. Sharing published material for non-commercial purposes is subject to the following conditions:

https://creativecommons. org//icenses/by-nc/4.0/legalcode sect. 3. Reproducing and sharing published material for commercial purposes is not allowed without permission in writing from the publisher. 
rate. ${ }^{2}$ Relapse after R-CHOP therapy occurs in $40 \%$ of patients; ${ }^{3,4}$ this is currently managed with salvage chemotherapy. This is followed by high-dose chemotherapy and autologous bone marrow transplant in patients with chemosensitive disease, which, however, leads to long-term disease control in only half of the patients. ${ }^{5}$ Moreover, less than $20 \%$ of patients treated with an R$\mathrm{CHOP}$ front-line regimen who relapse within one year benefit from salvage autologous hematopoietic cell transplant. ${ }^{2,6}$ Thus, novel therapeutic strategies that reduce relapse rates and enhance DLBCL patient survival are urgently needed.

Novel approaches based on selective-drug delivery to cancer cells promise to increase patient benefit by offering both higher cure rates and lower side effects in DLBCL patients. In this regard, we evaluated a previously developed protein nanocarrier as a possible drug carrier to pursue the selective elimination of DLBCL cells over-expressing CXCR4 (CXCR4 ${ }^{+}$), which are responsible for DLBCL relapse and disease progression. ${ }^{7-9}$ Thus, the CXCR4-CXCL12 axis is involved in tumor pathogenesis, cancer cell survival, stem cell phenotype, and resistance to chemotherapy. ${ }^{10,11}$ In addition, CXCR4 is constitutively over-expressed in NHL cell lines, ${ }^{12,13}$ and also in approximately $50 \%$ of malignant B-cell lymphocytes derived from DLBCL patients. ${ }^{8}$ Interestingly, CXCR $4^{+}$DLBCL cell lines show resistance to rituximab but are sensitive to the combination of rituximab with a CXCR4 antagonist. ${ }^{14,15}$ Most importantly, we and others reported that CXCR4 overexpression associates with poor progression-free and overall survival in DLBCL patients treated with R$\mathrm{CHOP}^{7,8,14}$

Our group has developed T22-GFP-H6, a self-assembling protein nanocarrier, which uses the peptidic T22 ligand to target the CXCR4 receptor. ${ }^{16}$ This carrier displays a high recirculation time in blood and selectively biodistributes to tumor tissues in solid tumor models, internalizing selectively in CXCR4 ${ }^{+}$cancer cells, while increasing its tumor uptake compared to the untargeted GFP-H6 counterpart. ${ }^{17}$ This nanocarrier is also able to incorporate toxins (e.g. diphtheria toxin catalytic domain) leading to selective elimination of CXCR4 ${ }^{+}$colorectal cancer cells. ${ }^{18,19}$ Nevertheless, no previous protein-based nanocarrier has been described to specifically target cancer cells in hematologic neoplasias. Critical differences between solid cancers and hematologic neoplasias may raise doubts about its use to target $\mathrm{CXCR} 4^{+}$cancer cells in DLBCL models. Thus, the enhanced permeability/retention (EPR) effect, due to abnormal fenestrated vessels and limited lymphatic drainage, allows nanocarrier accumulation in solid tumors. In contrast, DLBCL is a disseminated disease that displays freely circulating lymphoma cells in blood concomitantly with their confinement at specific tumor niches, such as lymph nodes (LN) and bone marrow (BM), in which the EPR effect is unlikely to be present. $^{20}$

Here, we studied whether active targeting of the T22GFP-H6 nanocarrier leads to its selective uptake in $\mathrm{CXCR}^{+}$subcutaneous (SC) DLBCL tumors. We also assessed if this increased uptake associates with specific nanocarrier internalization in CXCR4 $4^{+}$lymphoma cells; issues still be to settled in nanomedicine. ${ }^{21,22}$ Importantly, we used a disseminated CXCR $4^{+}$DLBCL model (which replicates the organ involvement observed in DLBCL patients ${ }^{8}$ ) to study nanocarrier accumulation in lym- phoma-affected organs (LN and BM) and its capacity to internalize in CXCR4 ${ }^{+}$lymphoma cells within these organs. Moreover, we evaluated whether T22-DITOXH6, a nanoparticle incorporating a diphtheria toxin domain that maintains the same structure as the nanocarrier, can selectively eliminate CXCR4 $4^{+}$DLBCL cells in SC tumors. The study goal was to determine whether we could use the nanocarrier to selectively deliver drugs to target CXCR4 ${ }^{+}$DLBCL cells.

\section{Methods}

\section{In vivo experiments}

Four-week old female NOD/SCID mice were obtained from Charles River Laboratories. Mice were maintained in specific pathogen-free (SPF) conditions with sterile food and water ad libitum. Mouse experiments were approved by the Hospital de la Santa Creu i Sant Pau Animal Ethics Committee.

For SC models, 10 million DLBCL cells were injected in both flanks. Tumor growth was monitored twice a week with a caliper (tumor volume $=$ width $^{2} \times$ length/2). When tumors reached a volume of $600-800 \mathrm{~mm}^{3}$, mice received a single intravenous (IV) dose of $200 \mu \mathrm{g}$ T22-GFP-H6, which contains a fluorescent domain, or buffer (20 $\mathrm{mM}$ Tris, $500 \mathrm{mM} \mathrm{NaCl}$, pH 8). T22-GFP-H6 design and production have been described in previous studies. ${ }^{16}$ Fluorescence intensity (FLI) was measured ex vivo at different time points in tumors, plasma, and all organs. A plasma pool was obtained by centrifugation of total blood, obtained by intracardiac puncture $(25 \mathrm{G})$, at $600 \mathrm{~g}$ for ten minutes (min) at $4^{\circ} \mathrm{C}$. T22-GFP-H6 biodistribution in SC tumors over time was measured using the area under the curve (AUC). AUC analysis of tumors and normal organs was measured using the GraphPad Prism 6 program. We subcutaneously administered AMD3100 in mice to perform CXCR4 blocking experiments, giving a total of three AMD3100 doses at $10 \mathrm{mg} / \mathrm{kg}, 1$ hour (h) before and $1 \mathrm{~h}$ and $2 \mathrm{~h}$ after IV T22-GFP-H6 injection. We used SC tumor models to evaluate the antitumor effect and associated toxicity of T22DITOX-H6. Mice received a single $25 \mu \mathrm{g}$ IV dose of T22DITOX-H6 or buffer when tumors reached a volume of 400-600 $\mathrm{mm}^{3}$. Animals were euthanized $24 \mathrm{~h}$ post administration. T22DITOX-H6 nanoparticle characterization has been published previously. ${ }^{18}$

To generate the disseminated lymphoma model, NOD/SCID mice were intravenously injected with $20 \times 10^{6}$ luminescent Toledo cells (Toledo-Luci) in $200 \mu \mathrm{L}$ physiological serum. Dissemination was monitored capturing bioluminescence intensity (BLI) twice a week after intraperitoneal injection of firefly D-luciferin. After 27-30 days, animals received a single IV dose of $400 \mu \mathrm{g}$ T22-GFP-H6 nanocarrier or buffer. Five hours later, FLI was measured ex vivo in all organs.

Fluorescence intensity correlates to the amount of accumulated protein in each tissue and is expressed as average radiant efficiency. FLI from experimental mice was calculated subtracting the FLI auto-fluorescence of control mice. The emitted FLI and BLI were measured using the IVIS Spectrum 200 Imaging System (Xenogen). Finally, tumors and all organs were collected, fixed and paraffined to perform histological, immunohistochemical or immunofluorescent evaluations, and were also directly cryopreserved in liquid nitrogen for protein extraction.

Details of methods for cell culture, transfection with Luciferase and CXCR4 plasmids, cell proliferation, flow cytometry, western blot, histopathology, 4',6-diamidino-2-phenylindole (DAPI) staining, immunohistochemistry (IHC) and immunofluorescence (IF) analyses can be found in the Online Supplementary Appendix. 


\section{Statistical analysis}

In vitro experiments were performed in biological triplicates while in vivo experiments were performed in triplicates/quadruplicates. The data for all experiments were reported as mean \pm Standard Error of Mean (SEM). All results were analyzed using the Student $t$-test. $P<0.05$ was considered statistically significant. Statistical calculations were performed using SPSS software version. $^{21}$

\section{Results}

CXCR4-dependent internalization of T22-GFP-H6 in human CXCR4 ${ }^{+}$diffuse large B-cell lymphoma cell lines

CXCR4 membrane levels were evaluated in four human DLBCL cell lines by flow cytometry (Figure 1A) and IHC (Online Supplementary Figure S1). CXCR4 expression was highest in Toledo cells, followed by U-2932 and RIVA, whereas CXCR4 expression in the SUDHL-2 cell line was undetectable. CXCR4-transfected SUDHL-2 cells (CXCR4+ SUDHL-2) showed average CXCR4 levels.

T22-GFP-H6 nanocarrier internalization correlated with CXCR4 expression. Thus, T22-GFP-H6 internalized the most in Toledo cells, followed by U-2932 and RIVA, whereas it did not internalize in SUDHL-2 (Figure 1B). Moreover, T22-GFP-H6 nanocarrier internalization was CXCR4-dependent. So, after preincubation with CXCR4 antagonist AMD3100, T22-GFP-H6 internalization decreased significantly in Toledo, U-2932 and RIVA cells (Figure 1C). As expected, T22-GFP-H6 did not internalize in SUDHL-2 cells (only background FLI was detected), whereas high internalization was registered in CXCR4 SUDHL-2 cells. Similarly, AMD3100 preincubation had no effect on nanocarrier internalization in SUDHL-2 cells but led to a significant decrease in CXCR4+ SUDHL-2 cells (Figure 1D). Thus, we showed specific in vitro entry of T22-GFP-H6 into CXCR4 ${ }^{+}$DLBCL cells through the CXCR4 receptor.

\section{Non-cytotoxic effect of T22-GFP-H6 in diffuse large \\ B-cell lymphoma cell lines in vitro}

After exposure to T22-GFP-H6 (50-500nM range), cell viability for all four evaluated DLBCL cell lines was approximately or above 100\% (Figure 1E). Therefore, T22-GFP-H6 nanocarrier has no in vitro antineoplastic effect against these DLBCL cell lines.
A

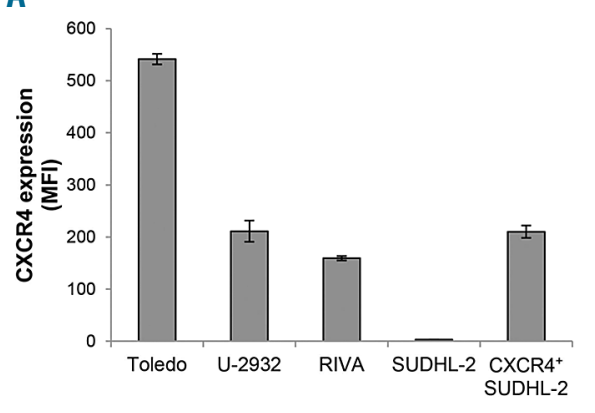

B

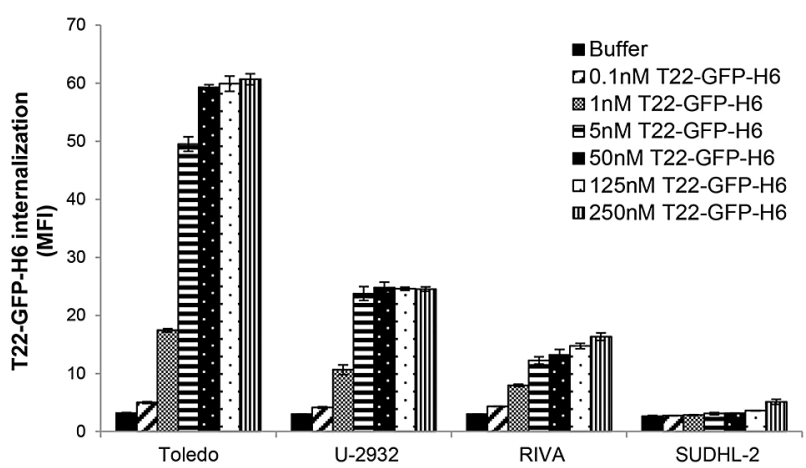

C

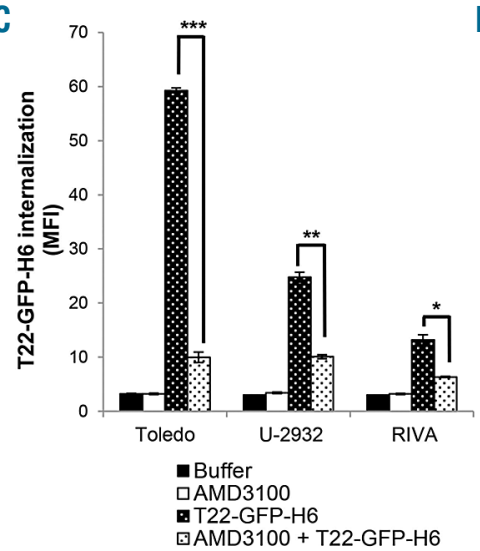

D

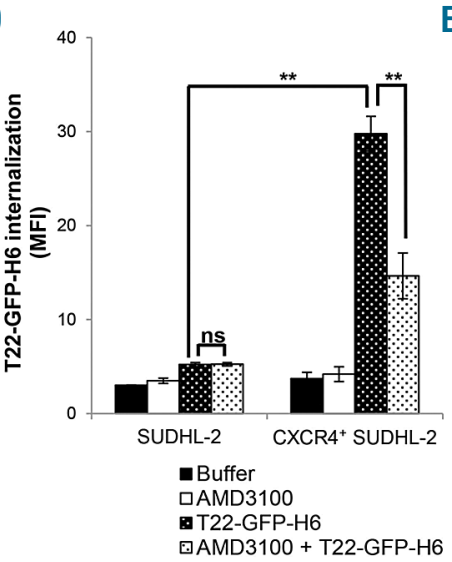

E

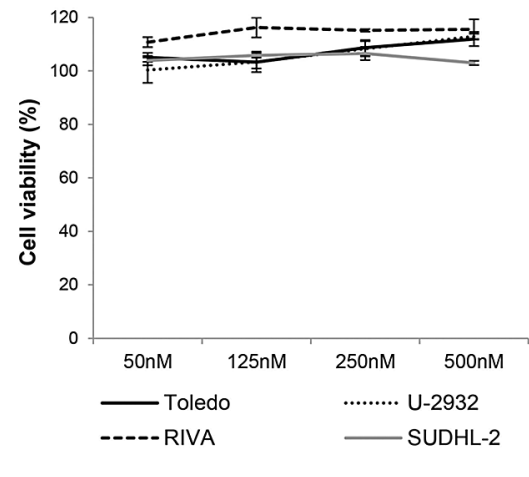

Figure 1. In vitro T22-GFP-H6 nanocarrier internalization in CXCR4 ${ }^{+}$diffuse large B-cell lymphoma (DLBCL) cell lines and its dependence on the CXCR4 receptor. (A) CXCR4 membrane expression of different DLBCL cell lines (Toledo, U-2932, RIVA and SUDHL-2) and the SUDHL-2 cell line transfected with a CXCR4 plasmid (CXCR4+ SUDHL-2) measured by flow cytometry. (B) Levels of intracellular fluorescence quantified by flow cytometry in Toledo, U-2932, RIVA and SUDHL-2 cells after 1 hour (h) exposure to T22-GFP-H6 nanocarrier at different concentrations (range: 0.1nM-250nM). (C) T22-GFP-H6 internalization, measured by flow cytometry, in Toledo, U-2932 and RIVA cells after $1 \mathrm{~h}$ pretreatment with the antagonist AMD3100 (50nM T22-GFP-H6:500nM AMD3100). (D) Competition assays with AMD3100 (250nM T22-GFP-H6: 2500nM AMD3100) in SUDHL-2 cells and CXCR4 ${ }^{+}$SUDHL-2 cells. (E) Lack of cytotoxicity (measured as percentage of cell viability) after 48h exposure to high concentrations of T22-GFP-H6 nanocarrier (range: 50nM-500nM) in Toledo, U-2932, RIVA and SUDHL-2 cells. *P<0.05; $* * P<0.01$; $* * * P<0.001$; ns: non-significant; MFI: mean fluorescence intensity. 
A

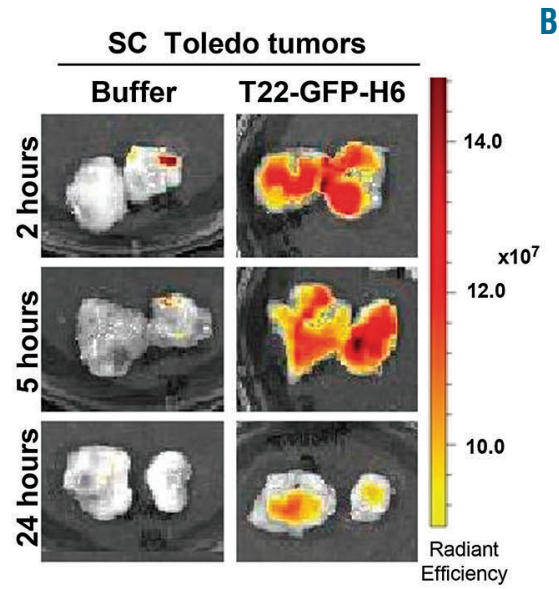

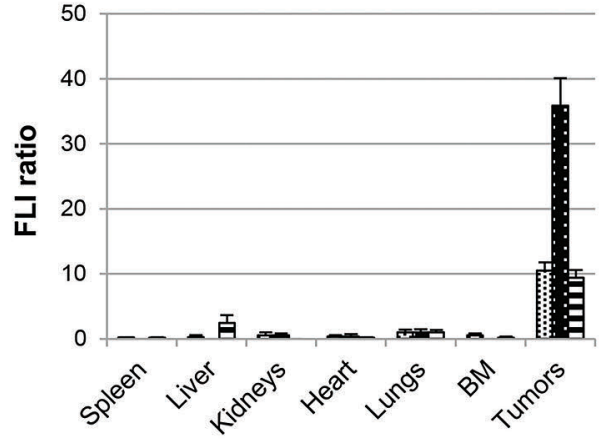

$\$ 2$ hours 5 hours $\$ 24$ hours

C

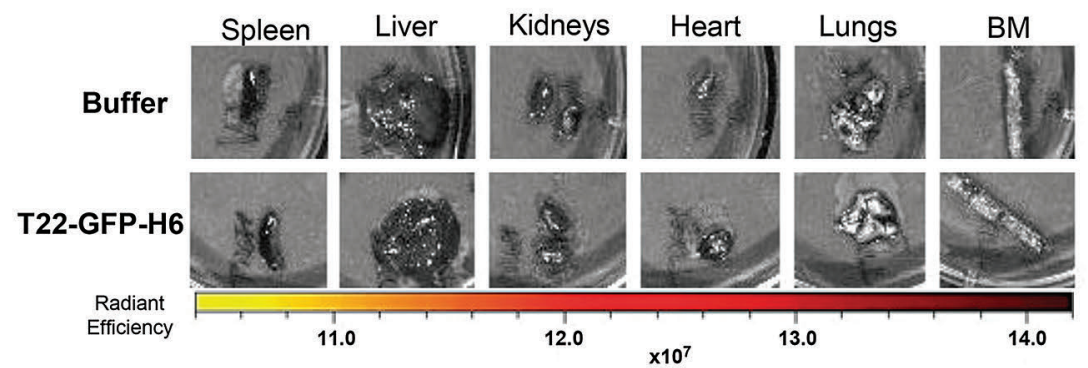

D

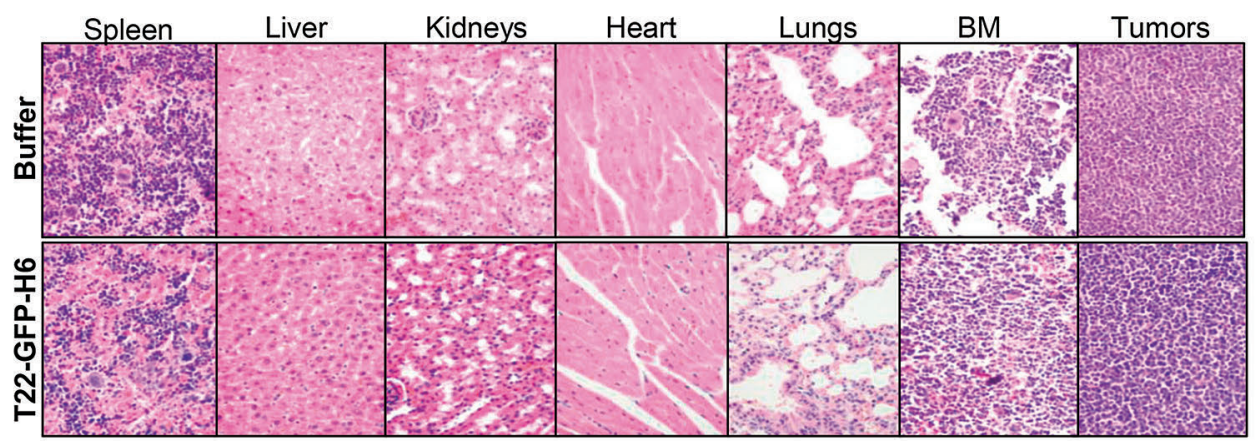

E

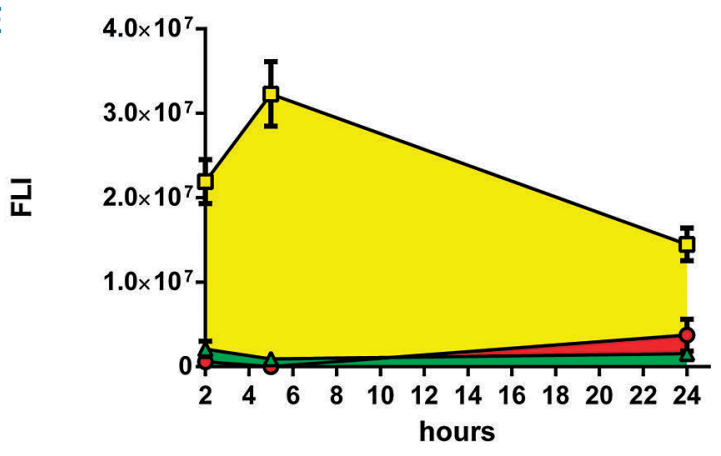

F

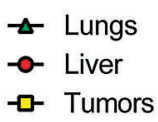

\begin{tabular}{|c|c|}
\cline { 2 - 2 } \multicolumn{1}{c|}{} & $\begin{array}{c}\text { T22-GFP-H6 } \\
\text { UPTAKE (\%) }\end{array}$ \\
\hline Spleen & $\mathbf{0 . 4 6} \pm 0.27$ \\
\hline Liver & $\mathbf{5 . 9 6} \pm 2.83$ \\
\hline Kidneys & $\mathbf{1 . 0 7} \pm 0.45$ \\
\hline Heart & $\mathbf{1 . 1 7} \pm 0.81$ \\
\hline Lungs & $\mathbf{4 . 5 5} \pm 1.20$ \\
\hline BM & $\mathbf{0 . 6 7} \pm 0.51$ \\
\hline Tumors & $\mathbf{8 6 . 1 3} \pm 4.04$ \\
\hline
\end{tabular}

Figure 2. In vivo biodistribution and toxicity assessment of T22-GFP-H6 in the CXCR4+ subcutaneous (SC) Toledo mouse model. (A) Fluorescence intensity (FLI) detection in SC Toledo tumors at 2, 5 or 24 hours (h) after intravenous (IV) injection of $200 \mu \mathrm{g}$ of T22-GFP-H6. No fluorescence was detected in buffer-treated mice. (B) Quantification of emitted fluorescence (measured as FLI ratio) at different times (2, 5 and $24 \mathrm{~h}$ ) in SC tumors and normal tissues (liver, spleen, heart, lungs, kidneys and bone marrow). (C) FLI emitted by the nanocarrier in normal tissues 5h after T22-GFP-H6 administration. (D) Hematoxylin \& Eosin (H\&E) stained tissue sections of normal organs and SC tumors at the $5 \mathrm{~h} \mathrm{FLI} \mathrm{peak.} \mathrm{(E)} \mathrm{Representation} \mathrm{of} \mathrm{the} \mathrm{area} \mathrm{under} \mathrm{the} \mathrm{curve} \mathrm{(AUC)} \mathrm{of} \mathrm{emitted} \mathrm{FLI} \mathrm{over} \mathrm{time} \mathrm{(2-24h)} \mathrm{registered} \mathrm{in} \mathrm{tumors,}$ liver and lungs in T22-GFP-H6-treated mice. Notice that the AUC in the organs with an uptake lower than $2 \%$ (spleen, kidney, heart and BM) is not visible in this graphic. (F) Percentage of nanocarrier uptake (as measured by the AUC of emitted FLI) registered in each organ studied in relation to the total emitted FLI (sum of AUC in tumors and all studied normal organs) during the $2-24 \mathrm{~h}$ period and expressed as mean \pm Standard Error of Mean (SEM). FLI ratio for experimental mice was calculated subtracting the FLI auto-fluorescence of control mice and dividing the FLI signal of each tumor/tissue by the FLI signal of the lungs (organ chosen as reference). Original magnification $\times 400$. BM: bone marrow. 
A

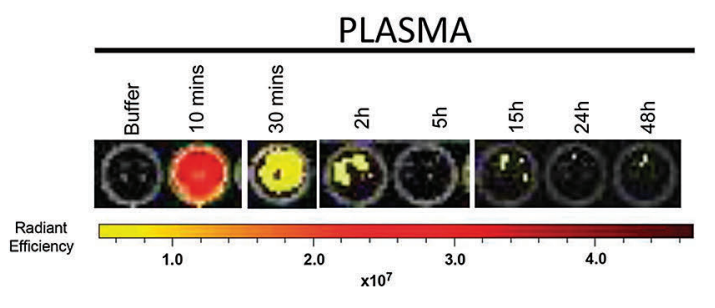

B

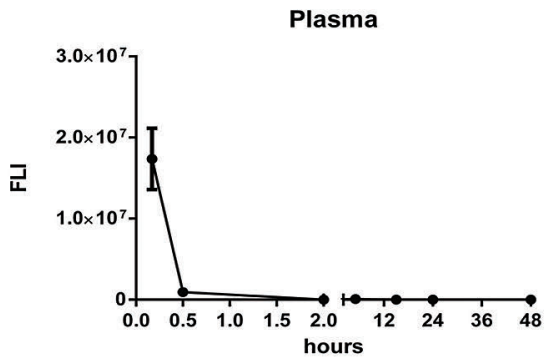

C

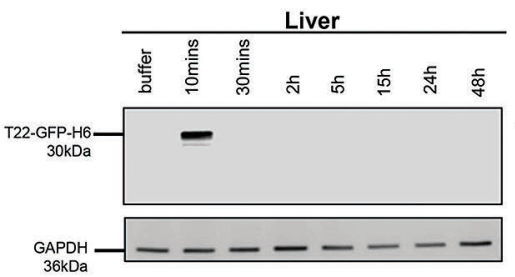

D

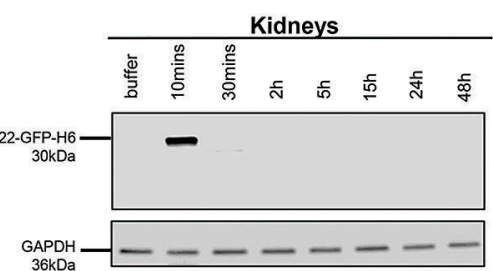

E

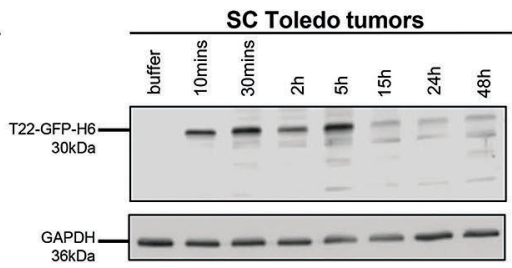

Figure 3. T22-GFP-H6 pharmacokinetics in plasma and clearance in CXCR4+ SC Toledo mouse model. (A) Representative images of fluorescence intensity (FLI) registered in plasma ( $250 \mu \mathrm{L}$ ) of mice treated with buffer or $200 \mu \mathrm{g}$ T22-GFP-H6, 10 minutes (min), $30 \mathrm{~min}, 2 \mathrm{hour}(\mathrm{h}), 5 \mathrm{~h}, 15 \mathrm{~h}, 24 \mathrm{~h}$ and $48 \mathrm{~h}$ after its administration. (B) Graphical representation of FLI quantification in plasma over time. (C and D) Western blot analysis of the fate of the T22-GFP-H6 protein in hepatic and renal tissue in mice treated with buffer or $10 \mathrm{~min}, 30 \mathrm{~min}, 2 \mathrm{~h}, 5 \mathrm{~h}, 15 \mathrm{~h}, 24 \mathrm{~h}$ and $48 \mathrm{~h}$ after nanocarrier administration. (E) Western blot analysis of the fate and processing of the T22-GFP-H6 protein in subcutaneous (SC) Toledo tumors in mice treated with buffer or $10 \mathrm{~min}, 30 \mathrm{~min}, 2 \mathrm{~h}, 5 \mathrm{~h}, 15 \mathrm{~h}, 24 \mathrm{~h}$ and $48 \mathrm{~h}$ after nanocarrier administration. Note the almost complete proteolysis of the full-length protein nanocarrier in tumor tissue, and the absence of proteolysis in liver and kidney. GAPDH was used as a loading control. T22-GFP-H6 was detected with an anti-GFP antibody.

Highly selective T22-GFP-H6 tumor uptake in mice bearing subcutaneous CXCR4+ ${ }^{+}$diffuse large B-cell lymphoma tumors without toxicity

We evaluated T22-GFP-H6 biodistribution in the CXCR4 4 SC Toledo mouse model, measuring the fluorescence emitted by the nanocarrier GFP domain, after a single $200 \mu \mathrm{g}$ IV dose. T22-GFP-H6 accumulated in CXCR4 ${ }^{+}$ SC tumors $2 \mathrm{~h}$ after injection, reaching a FLI peak $5 \mathrm{~h}$ post injection, and decreasing considerably after $24 \mathrm{~h}$ (Figure $2 \mathrm{~A}$ and $\mathrm{B})$. Tumor uptake $5 \mathrm{~h}$ post injection was 35.85 times higher than lung uptake, which was taken as a reference among the normal mouse organs because, although almost negligible, it did show the most sustained FLI emission over time (Figure $2 \mathrm{~B}$ and Online Supplementary Table S1). Similar observations were made in all non-tumor organs analyzed (Figure $2 \mathrm{~B}$ and $\mathrm{C}$ ). Moreover, we did not observe any histological alteration in Hematoxylin \& Eosin (H\&E) stained normal organs (Figure 2D).

The quantification of the AUC of emitted FLI over the study period (Figure 2E and Online Supplementary Table S2) showed that tumor tissue accumulated $86.13 \pm 4.04 \%$ of the total FLI detected in all organs, including tumor and non-tumor tissues. In contrast, the liver, which was the non-tumor organ with higher AUC, reached only $5.96 \pm 2.83 \%$ (Figure $2 \mathrm{~F}$ ). Therefore, T22-GFP-H6 displayed a specific targeting of CXCR4 ${ }^{+}$SC DLBCL tumors with negligible nanocarrier accumulation in non-tumor bearing organs, which supports a highly selective tumor uptake as compared to normal cells.

After a single T22-GFP-H6 IV administration, measurement of circulating nanocarrier showed a fast biodistribution half-life $\left(t_{1 / 2} \approx 20 \mathrm{~min}\right)$ in the blood compartment, followed by a slower elimination phase $\left(\mathrm{t}_{1 / 2} \approx 75 \mathrm{~min}\right)$, becoming undetectable in plasma at $2 \mathrm{~h}$ (Figure $3 \mathrm{~A}$ and $\mathrm{B}$ ).

The highly unusual T22-GFP-H6 tumor uptake and its low accumulation in the expected non-tumor drug clearance organs (i.e. liver and kidney) triggered the analysis of the nanocarrier fate in these organs by western blot. After a single T22-GFP-H6 dose, we observed the full-length protein $(\approx 30 \mathrm{kDa})$ present in liver and kidney $10 \mathrm{~min}$ post administration (Figure $3 \mathrm{C}$ and D), becoming undetectable over a period which ranged from $30 \mathrm{~min}$ to $48 \mathrm{~h}$. In sharp contrast, we detected full-length T22-GFP-H6 protein in Toledo SC tumors at $10 \mathrm{~min}, 30 \mathrm{~min}, 2 \mathrm{~h}$ and $5 \mathrm{~h}$. Interestingly, faint proteolytic bands appeared over a period which ranged from $30 \mathrm{~min}$ to $2 \mathrm{~h}$, which became more intense at $5 \mathrm{~h}$. Over a period which ranged from $15 \mathrm{~h}$ to $48 \mathrm{~h}$, the full-length protein decreased dramatically and the nananocarrier was mostly proteolyzed (Figure $3 \mathrm{E}$ ). These results, together with the observed FLI AUC in tumor and normal organs, suggest that the proteolytic activity observed in the tumor makes it the main nanocarrier clearance organ.

\section{T22-GFP-H6 and CXCR4 receptor co-localization in the cell membrane followed by its internalization in CXCR4+ diffuse large B-cell lymphoma cells}

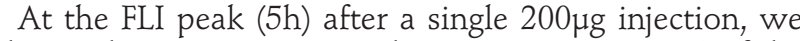
observed nanocarrier internalization in $56.2 \pm 12.0 \%$ of the Toledo cells (green staining with anti-GFP IF) in tumors, whereas all $(100 \%)$ tumor cells over-expressed CXCR4 (red staining with anti-CXCR4 IF). In buffer-treated tumors, the CXCR4 receptor localized mainly at Toledo cell membrane, while a dot-like staining inside the cell cytosol was observed in the nanocarrier-treated-tumors; a finding consistent with receptor internalization within endocytic vesicles. Merged (yellow) images showed nanocarrier and CXCR4 co-localization in the membrane of Toledo cells in T22-GFP-H6-treated tumors. Once into the cytosol, the CXCR4 and T22-GFP-H6 stained endoso- 


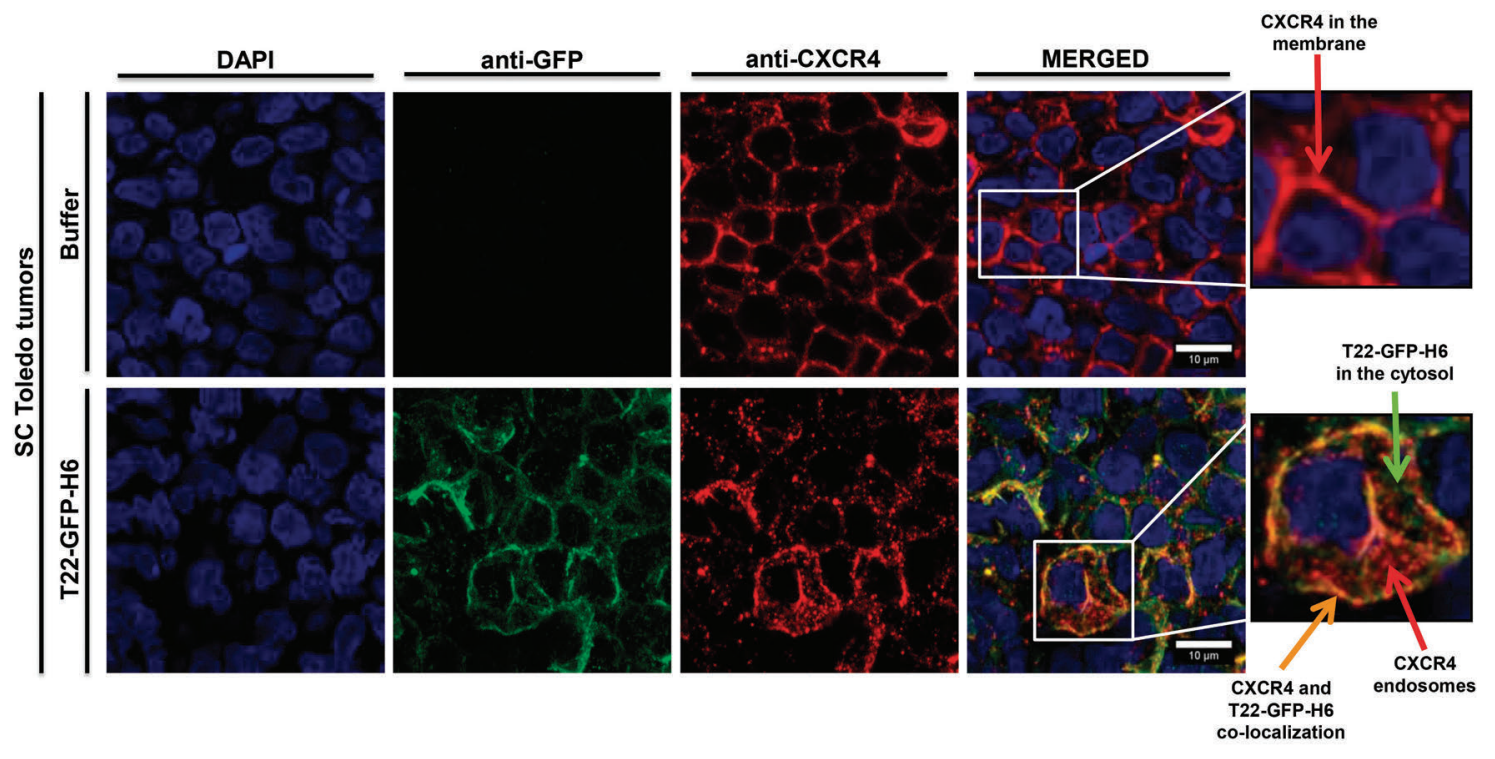

Figure 4. Co-localization of T22-GFP-H6 and CXCR4 receptor in the cell membrane and nanocarrier internalization in CXCR4 ${ }^{+}$subcutaneous (SC) tumors. Representative immunofluorescence images from SC Toledo tumors of mice treated with T22-GFP-H6 (200 $\mu$ g, 5h) or buffer. T22-GFP-H6 and CXCR4 co-localization was mainly seen in the cell membrane (yellow dots), whereas internalized nanocarriers were observed in the cell cytosol (green dots) and the endocytic vesicles with the CXCR4 receptor (red dots). DAPI staining (blue), anti-GFP protein (green), anti-CXCR4 receptor (red) and merged images from the three stains. Scale bars=10 $\mu \mathrm{m}$.

mal vesicles were dissociated (Figure 4). These results suggest that T22-GFP-H6 interacts with the CXCR4 receptor in the cell membrane, where both co-localize and, after internalizing jointly within endosomal vesicles, they are able to release the nanocarrier in the CXCR $4^{+}$DLBCL cell cytosol.

\section{Selective CXCR4-dependent T22-GFP-H6 tumor uptake} in subcutaneous diffuse large B-cell lymphoma tumors

We also assessed the dependence of nanocarrier tumor uptake on CXCR4 receptor, performing in vivo competition assays using the CXCR4 antagonist AMD3100 in mice bearing CXCR4 ${ }^{+}$Toledo-derived SC tumors (Figure $5 \mathrm{~A})$. Five hours after T22-GFP-H6 administration, we registered a peak of nanocarrier accumulation in tumors that reached 3.23 $\pm 0.38 \mathrm{E} 7$. In contrast, the AMD3100 administration prior and after nanocarrier injection blocked nanocarrier uptake in tumors, since the emitted FLI was 10 times lower $(0.31 \pm 0.52 \mathrm{E} 7)$ (Figure 5B). Differences between the Toledo tumors treated with T22-GFP-H6 and those treated with AMD3100 plus T22-GFP-H6 were highly significant (Figure 5C). This inhibition of nanocarrier uptake by AMD3100 confirms that tumor uptake depends on the CXCR4-receptor.

Additional support for this selective uptake comes from additional biodistribution assays comparing CXCR4 SUDHL-2 and CXCR4+ SUDHL-2 SC tumor-bearing mice. Five hours after $200 \mu \mathrm{g}$ T22-GFP-H6 administration, FLI emission from CXCR $4^{+}$SUDHL-2 tumors was significantly higher $(2.12 \pm 0.46 \mathrm{E} 7)$ than from CXCR4- SUDHL-2 tumors $(0.04 \pm 0.21 \mathrm{E} 7)$ (Figure $5 \mathrm{C}$ and D).

Consistently, Toledo and CXCR4 ${ }^{+}$SUDHL-2 tumors showed CXCR4 membrane expression, as measured by IHC, whereas CXCR4- SUDHL-2 tumors did not (Figure $5 \mathrm{E})$; a finding that confirms the specific directioning of T22-GFP-H6 to tumors containing CXCR4+ DLBCL cells.
T22-GFP-H6 biodistributes to all diffuse large B-cell lymphoma-infiltrated organs and internalizes in lymphoma cells in a CXCR4+ diffuse large B-cell lymphoma disseminated mouse model

We evaluated the biodistribution of T22-GFP-H6 in vivo in a CXCR4+ Toledo-Luci disseminated DLBCL mouse model, while monitoring lymphoma cell dissemination by measuring BLI levels emitted by the infiltrated organs in vivo (Figure 6A). In addition, we precisely identified the organs showing infiltration by Toledo-Luci cells, BM (cranium and hind limbs) and LN (cervical and renal). In some mice $(37.5 \%)$, we detected residual BLI levels in the spleen and no infiltration was observed in any other organ (Figure 6B). Macroscopic LN (cervical and renal) infiltration was identified in $100 \%$ of mice (Figure 6C). H\&E staining and anti-CD20 IHC confirmed Toledo-Luci cell infiltration in BM and LN tissue sections. CXCR4 membrane expression was maintained in DLBCL cells located in all infiltrated organs (Figure 6D).

We went on to study T22-GFP-H6 biodistribution after IV injection (400 $\mu \mathrm{g}$ dose) or buffer in mice displaying complete dissemination of Toledo cells (27-30 days post injection). Five hours after nanocarrier injection, we observed high FLI in BM (cranium and hind limbs) and LN (renal and cervical), whereas fluorescence was negligible or undetectable in non-infiltrated organs (Figure 7A and B). Indeed, T22-GFP-H6 was specifically delivered to the DLBCL infiltrated organs since FLI levels in BM and LN were 31.05- and 12.98-fold higher, respectively, in comparison to lungs (the reference organ showing background FLI levels) (Figure 7B and Online Supplementary Table S3). Moreover, no histopathological alterations were observed in any tissue analyzed in nanocarrier-treated mice (data not shown). IF analysis using anti-GFP showed T22-GFP-H6 (green) in Toledo-Luci cell cytosol in affected BM and LN. In addition, CXCR4 dot-like (red) and nanocarrier (green) 
staining co-localized (yellow) on the cell membrane. Moreover, similar to findings in SC Toledo tumors, in the disseminated model, we found a release of the nanocarrier into CXCR4 ${ }^{+}$DLBCL cell cytosol separated from endocytic vesicles containing the CXCR4 receptor (Figure 7C).
T22-GFP-H6 internalization in CXCR4+ mouse cells

To support the relevance of our CXCR $4^{+}$DLBCL models for clinical translation of the tumor (human cells) and nontumor (mouse cells) biodistribution data, we assessed whether the nanocarrier internalized in mouse cells.
A

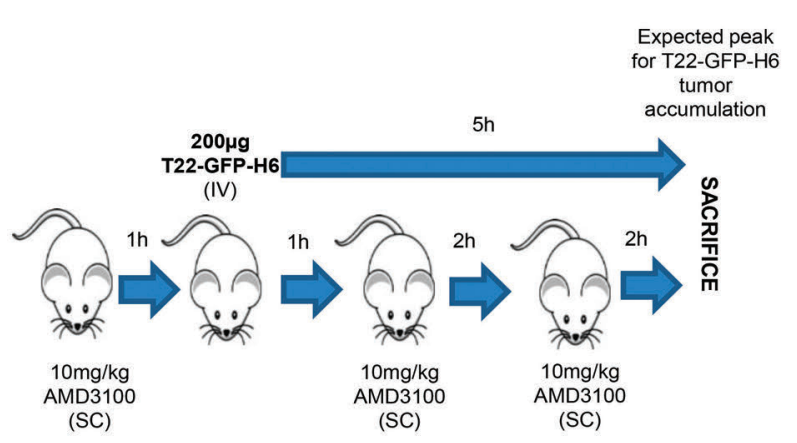

C

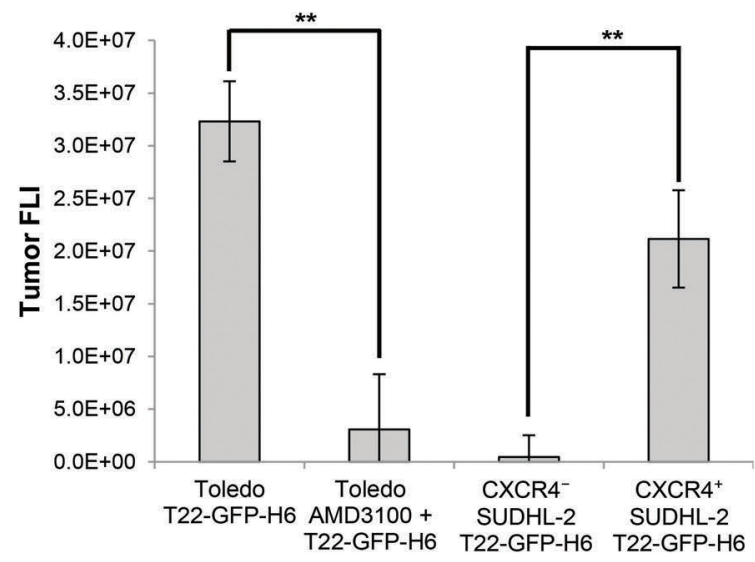

B

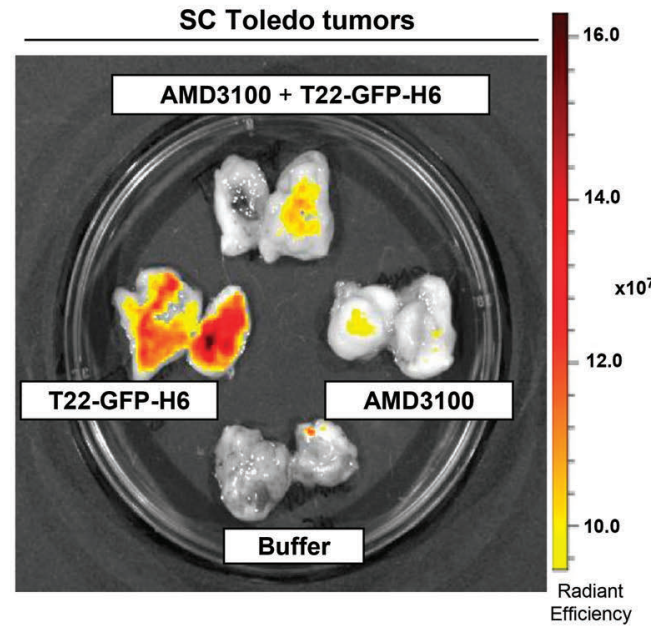

D

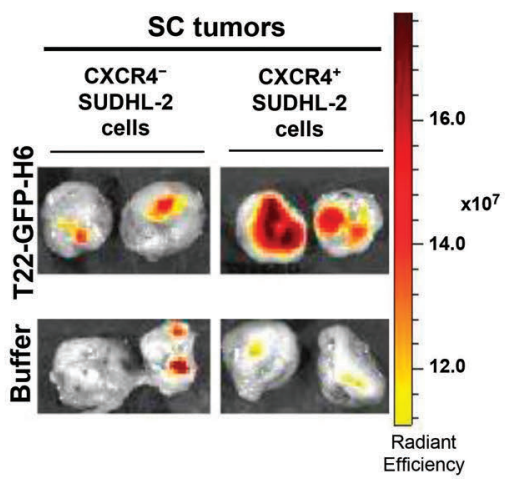

$\mathbf{E}$

CXCR4 expression (IHC)

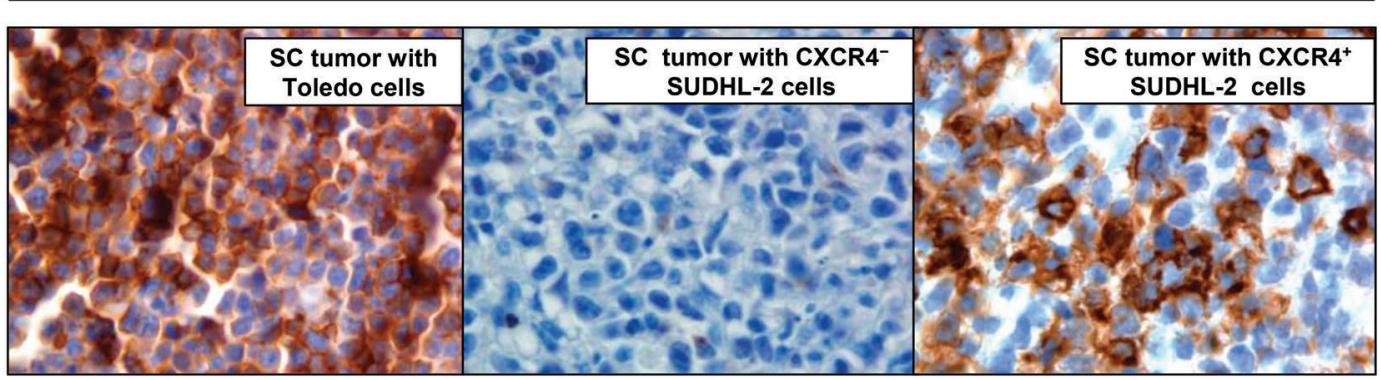

Figure 5. CXCR4-dependent uptake of T22-GFP-H6 nanocarrier in diffuse large B-cell lymphoma (DLBCL) subcutaneous (SC) tumors. (A) For the CXCR4 blocking experiment, mice were injected with a total of three SC doses of AMD3100 at $10 \mathrm{mg} / \mathrm{kg}$. The time point of mice sacrifice was 2 hours (h) after the last AMD3100 SC injection, which corresponds to the $5 \mathrm{~h}$ fluorescence intensity (FLI) peak after T22-GFP-H6 injection. (B) Representative images of emitted FLI by SC Toledo tumors from buffer, T22-GFP-H6, AMD3100 or AMD3100+T22-GFP-H6 treated animals. (C) FLI levels of SC tumor-bearing-mice of Toledo cells administered with T22-GFPH6 or AMD3100+T22-GFP-H6 and FLI levels of the tumors in T22-GFP-H6-treated bearing SC CXCR4+ SUDHL-2 tumors or SC CXCR4- SUDHL-2 tumors. (D) A representative image of the FLI in SC tumors of CXCR4- SUDHL-2 and CXCR4 ${ }^{+}$SUDHL-2 cells after $5 \mathrm{~h}$ of T22-GFP-H6 or buffer administration. (E) Level of membrane CXCR4 expression detected by immunohistochemistry (IHC) in SC tumors derived from Toledo, CXCR4 ${ }^{+}$SUDHL-2 and CXCR4 ${ }^{-}$SUDHL-2 cells. **P<0.01. Original magnification x1000. 
Firstly, we evaluated CXCR4 expression in the mouse B-cell lymphoma WEHI-231 cell line that showed medium CXCR4 membrane expression by flow cytometry and IHC (Online Supplementary Figure S2A). Then, we demonstrated intracellular nanocarrier uptake in mouse WEHI231 cells and its dependence on CXCR4 expression, since it was inhibited by AMD3100 (Online Supplementary Figure $S 2 B)$. Therefore, T22-GFP-H6 internalizes in both CXCR4 $4^{+}$human and CXCR4 $4^{+}$mouse lymphoma cells.
T22-DITOX-H6 antitumor effect and lack of toxicity in a CXCR4+ subcutaneous diffuse large B-cell lymphoma mouse model

Finally, we evaluated whether the therapeutic nanoparticle T22-DITOX-H6, incorporating a toxin domain with known antitumor activity, induced cell death of Toledo cells in SC tumors without damaging normal cells. T22DITOX-H6 caused apoptosis in lymphoma cells in these tumors since a single IV $25 \mu \mathrm{g}$ T22-DITOX-H6 dose signif-

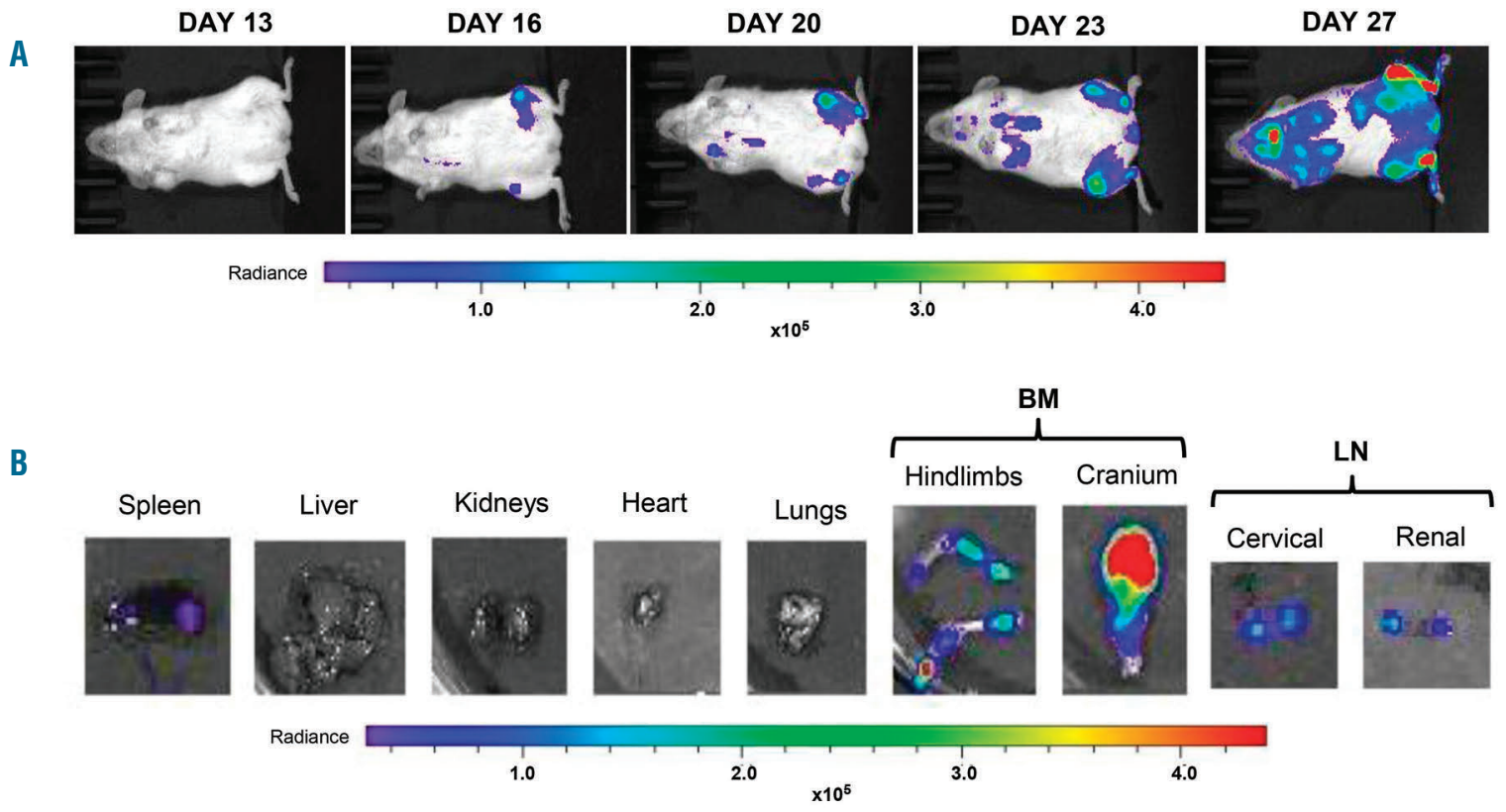

C

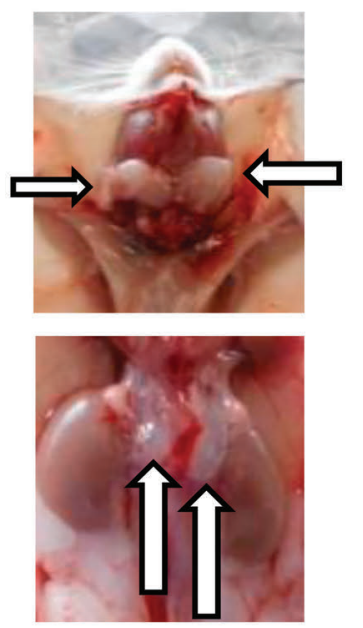

D

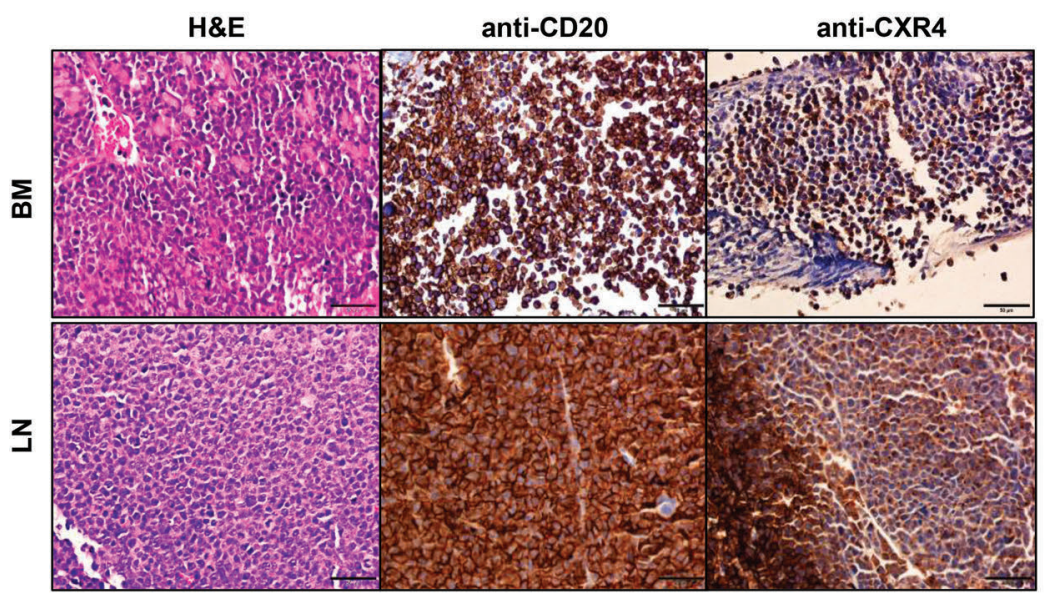

Figure 6. Pattern of organ infiltration in the Toledo-Luci diffuse large B-cell lymphoma (DLBCL) disseminated mouse model. (A) Bioluminescent intensity (BLI) follow up by IVIS Spectrum of mice intravenously injected with Toledo cells transfected with the Luciferase gene (Toledo-Luci cells). (B) Ex vivo representative images of the recorded BLI emission in different mouse organs: spleen, liver, kidneys, heart, lungs, hind limbs, cranium, cervical lymph nodes (LN) and renal LN. (C) Macroscopic images showing Toledo-Luci cell infiltration in cervical LN and renal LN. White arrows show the LN location. (D) Hematoxylin \& Eosin (H\&E) staining, anti-CD20 immunohistochemistry (IHC) for B-cell detection, and anti-CXCR4 IHC in bone marrow (BM) (cranium) and LN (cervical). Original magnification x400. Scale bars $=50$ $\mu \mathrm{m}$. 
icantly increased the number of apoptotic bodies and cleaved PARP level compared to buffer-treated mice (Figure $8 \mathrm{~A}$ and $\mathrm{B})$.

We then confirmed CXCR4 expression in hematopoietic cells of the mouse BM (CXCR4 ${ }^{+}$CD20- staining) (Figure
8C). A direct comparison showed that CXCR4 expression in SC Toledo tumors was significantly (22.87 times) higher than CXCR4 in mouse BM hematopoietic cells (Online Supplementary Figure S2C and D). No histopathological alterations (H\&E) nor induction of cell death (DAPI stain-
A

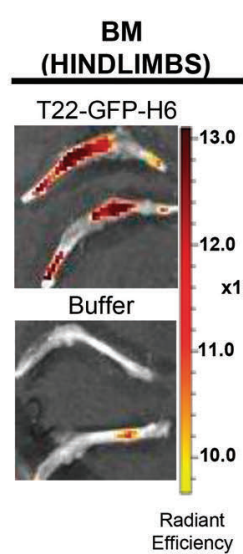

BM (CRANIUM)

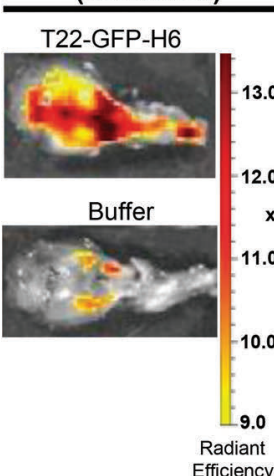

CERVICAL

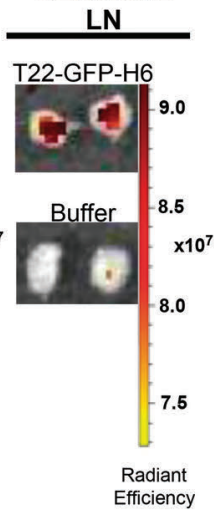

B

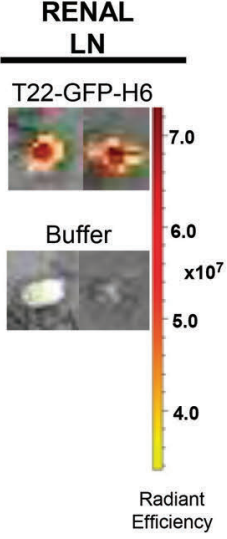

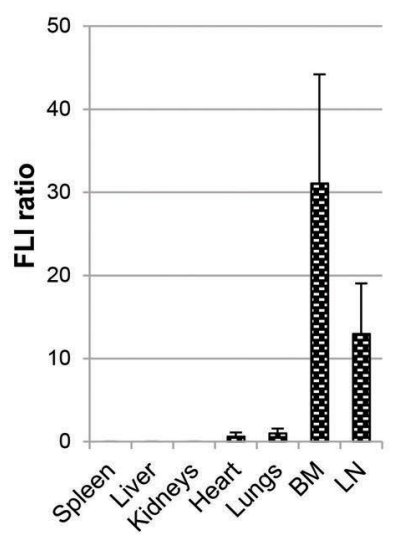

C
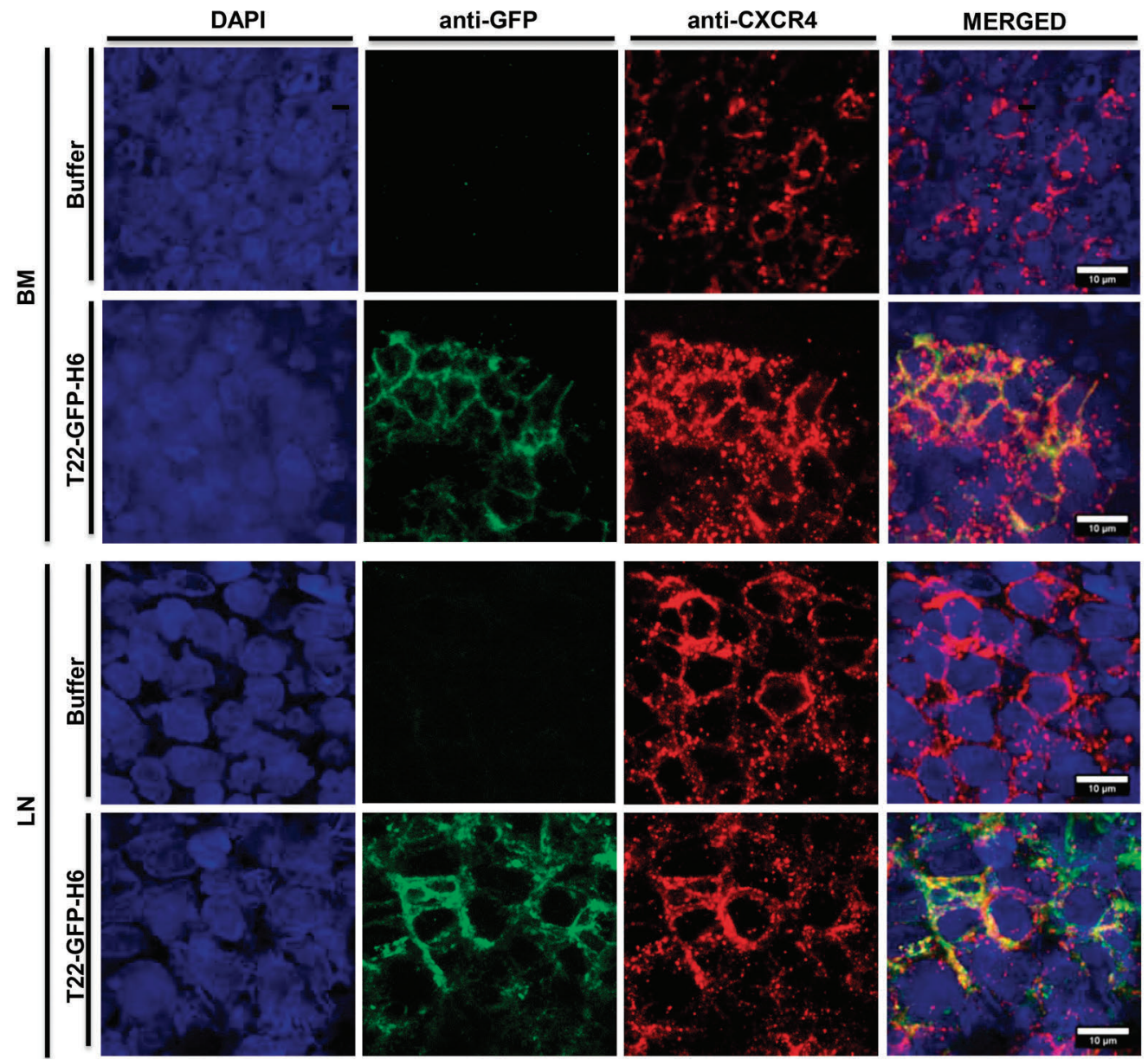

Figure 7. T22-GFP-H6 nanocarrier biodistribution in the Toledo-Luci diffuse large B-cell lymphoma (DLBCL) disseminated mouse model. (A) Level of fluorescence intensity (FLI) emission in bone marrow (BM) (cranium and hind limbs) and lymph nodes (LN) (cervical and renal) 5 hours (h) after the administration of $400 \mu \mathrm{g}$ T22GFP-H6 or buffer in a Toledo-Luci disseminated mouse model. (B) Comparison of FLI emission by T22-GFP-H6 accumulated in infiltrated DLBCL organs (BM and LN) as compared to non-DLBCL infiltrated organs (spleen, liver, kidneys, heart and lungs). FLI ratio from experimental mice was calculated subtracting the FLI auto-fluorescence of control mice and dividing the FLI recorded for each tissue by the FLI emitted by the lungs. (C) Representative immunofluorescent images of BM (cranium) and LN (cervical) in nanocarrier-treated mice and buffer-treated mice. Notice that green dots depicting internalized nanocarrier in the cytosol are only observed in T22-GFP-H6 treated animals. DAPI staining (blue), anti-GFP protein (green), anti-CXCR4 receptor (red) and merged images from the three stains. Scale bars=10 $\mu \mathrm{m}$. 
ing) was observed in the BM of T22-DITOX-H6-treated mice (Figure 8C). Lastly, we did not find any macroscopic (data not shown) or microscopic (H\&E staining) alteration in liver and kidneys (Figure 8D). Our results support the use of the nanocarrier under examination to efficiently deliver antitumor agents to achieve the selective killing of CXCR4 lymphoma cells without inducing toxicity on CXCR4 $4^{+}$ mouse hematopoietic cells or systemic organs.
A
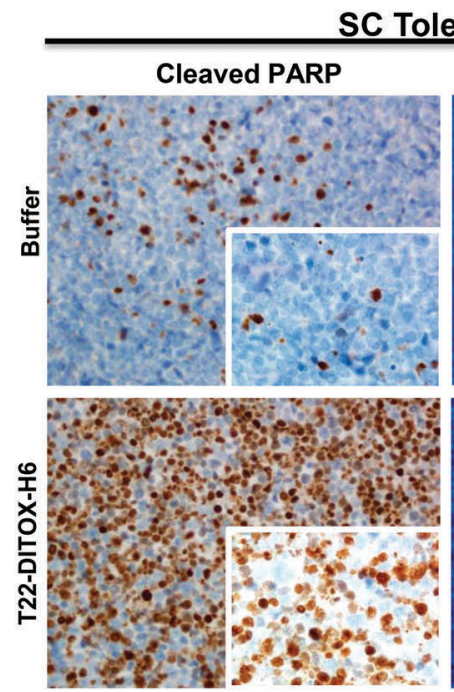

C
B

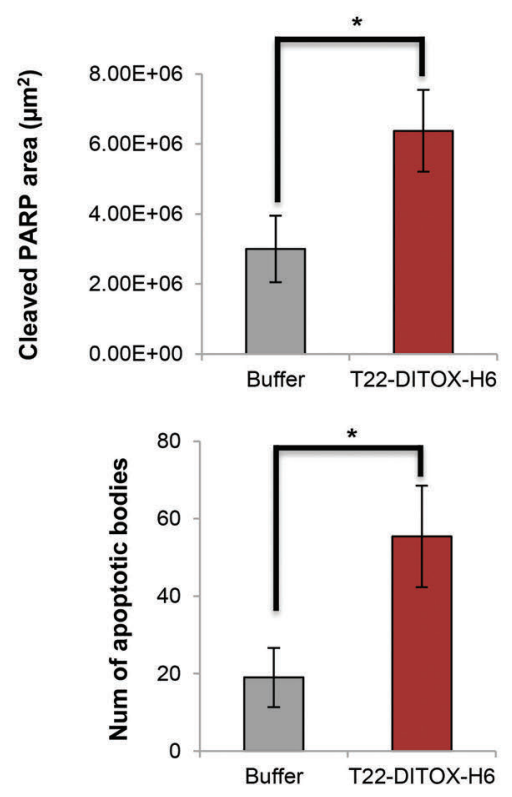

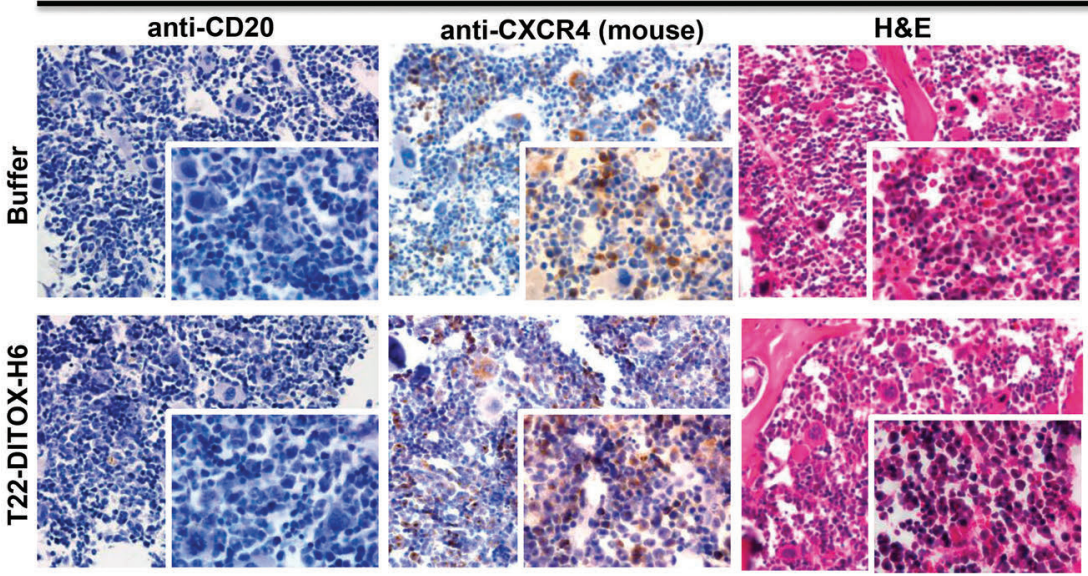

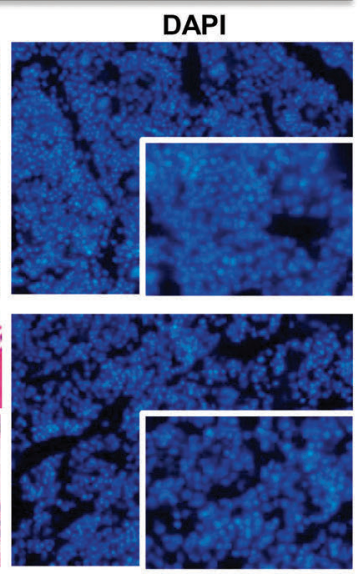

D

Liver

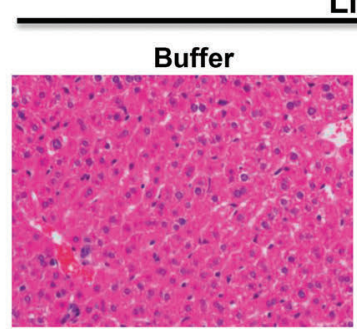

BM

DAPI
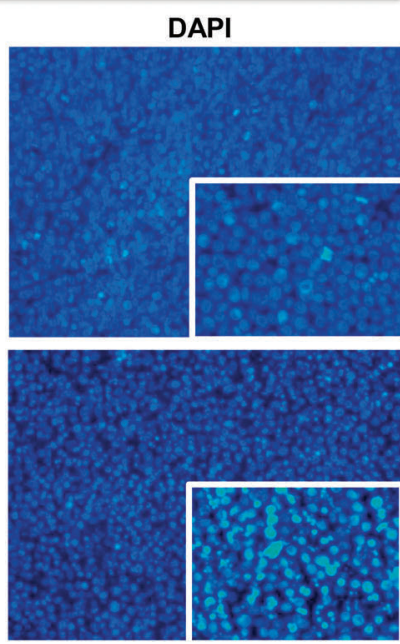

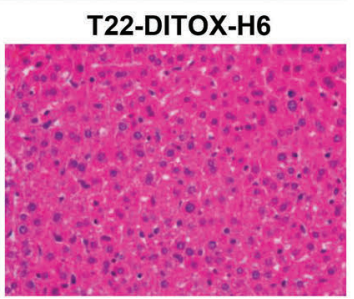

Kidneys

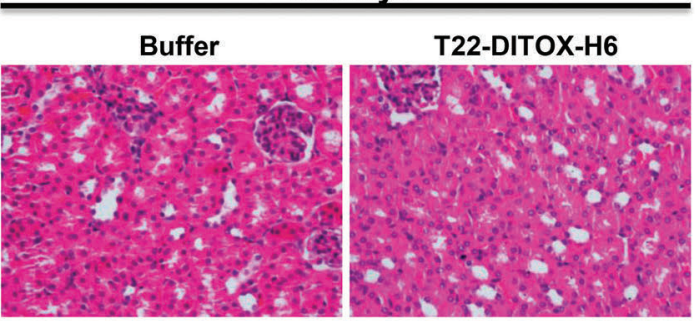

Figure 8. Evaluation of T22-DITOX-H6 antitumor effect and toxicity in a CXCR4 ${ }^{+}$subcutaneous (SC) Toledo mouse model. (A) Apoptosis detection by cleaved Poly(ADP-ribose) polymerase (PARP) immunohistochemistry (IHC) as well as nuclear condensation by 4',6-diamidino-2-phenylindole (DAPI) staining. (B) Mean quantification of cleaved PARP stained area (above) and number of apoptotic bodies by DAPI staining (bottom) in SC Toledo tumors 24 hours (h) after treatment with buffer or a single intravenous (IV) injection of $25 \mu \mathrm{g}$ T22-DITOX-H6. (C) Lack of T22-DITOX-H6-induced toxicity in mouse bone marrow (BM). Human anti-CD20 and mouse anti-CXCR4 IHC assays were used to identify CXCR4+ mouse cells resident in BM. Hematoxylin \& Eosin (H\&E) and DAPI staining samples were examined to detect possible alterations in cell morphology or cell death induction. (D) Lack of systemic toxicity in liver or kidney by histological analysis of tissue sections H\&E $24 \mathrm{~h}$ after treatment with buffer or $25 \mu \mathrm{g}$ T22-DITOX-H6. All images were taken at x400 and inserts at x1000. $* P<0.05$. 


\section{Discussion}

A huge limitation for the clinical translation of nanomedicines in oncology is the fact that only $0.7-5.0 \%$ of the administered dose reaches the tumor. ${ }^{23,24}$ In contrast, our biodistribution studies show a very high level of T22GFP-H6 uptake in tumor tissue (86.1\% of the total emitted fluorescence) compared to the combined fluorescence emitted by all normal tissues (13.9\% of total tumor+nontumor fluorescence), including the spleen, liver, kidney, heart, lung and BM. We have recently reported a similar finding for the same nanocarrier in a SC colorectal cancer (CRC) model. ${ }^{25}$ These data are consistent with the fast biodistribution half-life for the nanocarrier in blood (approx. $20 \mathrm{~min}$ ) and the detection of the full length protein in the $10 \mathrm{~min}-5 \mathrm{~h}$ period in SC CXCR $4^{+}$DLBCL tumors. Unexpectedly, we found that most of the proteolytic metabolism of T22-GFP-H6 occurs in tumor tissues, whereas clearance in liver or kidney is negligible, being detectable in these organs at $10 \mathrm{~min}$, probably by accessing the fenestrated vessels during a short time period, but being unable to reach their parenchyma. Our data are in dramatic contrast to the reported biodistribution of most nanocarriers studied so far, regardless of whether this was targeted actively or passively.

Nowadays, most nanocarriers that transport medicinal drugs in clinical trials, or that are available on the market, use passive targeting (e.g. liposomal doxorubicin or albumin-paclitaxel). They enhance the drug antitumor effect because its particulate size increases its permeability and retention in the tumor (EPR effect). Nevertheless, $50-80 \%$ of these nanocarriers accumulate in the liver. ${ }^{26}$ Although still at an initial stage, active nanocarrier targeting is being developed to selectively deliver antitumor drugs to tumor cells through specific surface receptors. ${ }^{27}$ Regarding B-cell lymphoma therapy, the use of doxorubicin-loaded mesoporous silica nanoparticles bound to rituximab, for targeting $\mathrm{CD} 20^{+} \mathrm{B}$ cells, demonstrated a significant increase in doxorubicin tumor uptake and higher inhibition of tumor growth than free doxorubicin. ${ }^{28}$ Moreover, additional targeted and non-targeted therapeutic nanoparticles are currently being evaluated for treatment of B-cell malignancies; however, no efficacy data are available yet because Phase I clinical assays to test their tolerability are still ongoing. ${ }^{29}$

The strategy we have used here with the actively targeted T22-GFP-H6 nanocarrier achieves selective and enhanced biodistribution to tumor tissue with no toxicity in the non-tumor organs. One possible explanation for the enhanced T22-GFP-H6 tumor uptake relates to the nature of the nanocarrier material. While our nanocarrier is made of self-assembled proteins, most, if not all, nanocarriers showing limited biodistribution to tumor are either inorganic (gold, silica, iron oxide, quantum dots) or organic (dendrimers, liposomes polymers, hydrogels) rather than protein-based. ${ }^{23,24}$ Once administered in blood, non-protein-based nanocarriers are covered by a protein corona that changes the conformation of the nanocarrier surface $e^{30}$ and undergo intensive phagocytosis by resident macrophages in clearance organs. ${ }^{31}$ A completely different protein drug delivery system is represented by the targeted antibody-drug conjugates (ADC), which have lower loading capacity and flexibility for encapsulating various cargos and display a less controllable drug release kinetics compared to nanocarriers. ${ }^{32}$ Consequently, in clinical studies, only $0.001-0.01 \%$ of the injected antibody dose reaches the tumor ${ }^{33}$ thus, although $\mathrm{ADC}$ are standard treatment in some neoplasias, protein nanocarriers could offer an enormous opportunity to improve drug delivery to tumors.

Our results on nanocarrier biodistribution in the SC tumor model demonstrate a specific co-localization of the nanocarrier together with the CXCR4 receptor in the cell membrane followed by their internalization, via endocytosis, to reach the cytosol of CXCR4 $4^{+}$DLBCL cells. Once inside the cytosol, the structure of the nanocarrier elicits endosomal escape and delivery of the materials into the cytoplasm, before its ultimate intracellular proteolysis. ${ }^{16}$

Furthermore, the efficacy of a T22-GFP-H6 nanocarrier that targets CXCR4 $4^{+}$DLBCL cells appears to be exclusively dependent on the overexpression of CXCR4 receptor in the membrane of tumor cells. This notion is currently supported by two main findings: on the one hand, T22-GFPH6 displays a tumor uptake significantly higher than that achieved in the same SC tumor when CXCR4 is inhibited by AMD3100 in the competition assay. On the other hand, T22-GFP-H6 administration to mice bearing CXCR4 $4^{+}$SC SUDHL-2 tumors shows significantly higher uptake than CXCR4- SC SUDHL-2 tumors. Moreover, we confirmed the capacity of T22-GFP-H6 to internalize in $\mathrm{CXCR}^{+}$mouse cells, similar to our findings in CXCR4 $4^{+}$ human cells. Thus, the high T22-GFP-H6 tumor uptake, and its low uptake in non-tumor organs, is necessarily related to the huge CXCR4 overexpression in DLBCL lymphoma cells and the negligible or low CXCR4 expression in normal organs, including BM mouse hematopoietic cells.

Importantly, in the disseminated CXCR4 $4^{+} \mathrm{DLBCL}$ mouse model, involving BM and LN, this nanocarrier also shows a high tumor uptake in the organs affected by CXCR $4^{+}$lymphoma cells, while displaying low biodistribution to normal tissues (with low or null CXCR4 expression). Unlike low molecular weight drugs that passively diffuse to all cells in the body, the biodistribution of the nanocarrier, or drug-loaded nanocarriers, is limited by their size; thus, it becomes highly dependent on the physiology and anatomy of specific organs in the body. Nanocarriers are unable to access organs irrigated by vessels with continuous endothelia and unable to penetrate membranes, unless they are actively targeted for endocytosis. ${ }^{34}$ Our protein nanocarrier can accumulate in the sinusoids of BM and LN infiltrated with tumor cells because they display vascular beds with discontinuous endothelium and 100-200nm fenestrations that allow the transport of macromolecules, including nanocarriers. ${ }^{35-37}$ Moreover, as we have showed in the SC mouse model, T22-GFP-H6 also has the capacity to internalize specifically in the CXCR4 $4^{+}$DLBCL cells, here localized in BM and LN in the DLBCL disseminated model. Even though there is no consistent EPR effect in hematologic neoplasias ${ }^{20}$ the structure of the vessels in the sinusoids of the DLBCL niches and the active targeting to CXCR4 allow T22-GFP-H6 accumulation and internalization in the tumor niches that are infiltrated by CXCR4+ DLBCL cells.

Given the high selectivity that T22-GFP-H6 achieves in targeting CXCR $4^{+}$DLBCL cells within the tumor, we used the SC CXCR4+ Toledo model to test the antitumor activity of T22-DITOX-H6, a therapeutic nanoparticle derived from this nanocarrier that incorporates the diphtheria cytotoxic domain. This therapeutic nanoparticle induced a high level of apoptotic cell death in tumor tissue without toxicity, since it did not induce any macroscopic or histo- 
logical alteration in normal organs, including the BM. The higher levels of CXCR4 expression in DLBCL cells, as compared to normal hematopoietic cells in the BM, were likely responsible for the cytotoxic activity, observed exclusively in tumor cells. These data confirm the capacity of the studied protein nanocarrier to be used as a platform for the delivery of antitumor agents to DLBCL cells. We have previously described also the potential use of T22GFP-H6 as an antitumor drug delivery agent for the treatment of colorectal cancer and leukemia. ${ }^{18,38,39}$ To our knowledge, no protein-based therapeutic nanoparticle has been previously reported as a possible drug carrier for lymphoma therapy.

So far, most research studies for DLBCL therapy targeting CXCR4 are performed with CXCR4 antagonists (e.g. plerixafor or BKT140) $)^{8,10,15}$ or inverse agonists (e.g. IOS01.01RS)..$^{40}$ Our approach differs from these studies since it is not focused on inhibiting signaling downstream of the CXCR4 receptor, but, instead, in delivering high concentrations of potent therapeutic agents to specifically kill CXCR4 $4^{+}$lymphoma cells. The active delivery of the drugloaded nanocarriers only to $\mathrm{CXCR} 4^{+}$cells should increase the therapeutic index compared to low molecular weight CXCR4 inhibitors, which biodistribute to all tissues independently of their CXCR4 expression. ${ }^{41,42}$ In conclusion, specifically eliminating CXCR $4^{+}$DLBCL cells could be an effective strategy to enhance the survival and cure rates observed in R-CHOP refractory or relapsed patients.

\section{Acknowledgments}

The authors would like to thank Annabel Garcia-León (IIB-
Sant Pau, Barcelona) and Lola Mulero Pérez (histology unit from $C M R B$, Barcelona) for their technical support.

\section{Funding}

This work was supported by Instituto de Salud Carlos III (ISCIII, Co-funding from FEDER) [PI18/00650, PIE15/00028, PI15/00378 and EU COST Action CA 17140 to RM, FIS PI17/01246, RD12/0036/0071 and FIS PI14/00450 to JS; CP15/00163 to MVC; FIS PI15/00272 to EV]; Agencia Estatal de Investigación (AEI) and Fondo Europeo de Desarrollo Regional (FEDER) (grant BIO2016-76063-R, AEI/FEDER, UE) to $A V$; CIBER-BBN [CB06/01/1031 and 4NanoMets to $R M$, and VENOM4CANCER to AV]; AGAUR [2017 FI_B 00680 to $A F$; 2017-SGR-865 to RM, 2017-SGR-1395 to JS and 2017SGR-229 to AV]; Josep Carreras Leukemia Research Institute [P/AG to RM]; a grant from the Cellex Foundation, Barcelona [to JS]; a grant from La Generalitat de Catalunya (PERIS) [SLTO02/16/00433 to JS]; a grant from Fundacion MMA [AP166942017 to MVC] and the Generalitat de Catalunya CERCA Programme. The work was also funded by Grants PERIS SLT006/17/00093 [to UU], Fundación Española de Hematología y Hemoterapia (FEHH) [to VP] and a Miguel Servet contract from ISCIII to MVC. The bioluminescent followup of cancer cells and nanoparticle biodistribution and toxicity studies have been performed in the ICTS-141007 Nanbiosis Platform, using its CIBER-BBN Nanotoxicology Unit (http://www.nanbiosis.es/portfolio/u18-nanotoxicology-unit/). Protein production has been partially performed by the ICTS "NANBIOSIS", more specifically by the Protein Production Platform of CIBER-BBN/ IBB (http://www.nanbiosis. es/unit/u1-protein-production-platform-ppp/).

\section{References}

1. Campo E, Swerdlow SH, Harris NL, Pileri S, Stein H, Jaffe ES. The 2008 WHO classification of lymphoid neoplasms and beyond: evolving concepts and practical applications. Blood. 2011;117(19):50195032.

2. Nowakowski GS, Blum KA, Kahl BS, et al. Beyond RCHOP: A Blueprint for Diffuse Large B Cell Lymphoma Research. J Natl Cancer Inst. 2016;108(12)

3. Cultrera JL, Dalia SM. Diffuse large B-cell lymphoma: current strategies and future directions. Cancer Control J Moffitt Cancer Cent. 2012;19(3):204-213.

4. Feugier P, Van Hoof A, Sebban C, et al. Long-term results of the R-CHOP study in the treatment of elderly patients with diffuse large B-cell lymphoma: a study by the Groupe d'Etude des Lymphomes de l'Adulte. J Clin Oncol. 2005;23(18):41174126.

5. Philip T, Guglielmi C, Hagenbeek A, et al. Autologous bone marrow transplantation as compared with salvage chemotherapy in relapses of chemotherapy-sensitive nonHodgkin's lymphoma. N Engl J Med. 1995;333(23):1540-1545.

6. Gisselbrecht C, Glass B, Mounier N, et al. Salvage regimens with autologous transplantation for relapsed large B-cell lymphoma in the rituximab era. J Clin Oncol. 2010;28(27):4184-4190.

7. Chen J, Xu-Monette ZY, Deng L, et al.
Dysregulated CXCR4 expression promotes lymphoma cell survival and independently predicts disease progression in germinal center B-cell-like diffuse large B-cell lymphoma. Oncotarget 2015;6(8):5597-5614.

8. Moreno MJ, Bosch R, Dieguez-Gonzalez R, et al. CXCR4 expression enhances diffuse large $\mathrm{B}$ cell lymphoma dissemination and decreases patient survival. J Pathol. 2015; 235(3):445-455.

9. Xu Z-Z, Shen J-K, Zhao S-Q, Li J-M. Clinical significance of chemokine receptor CXCR4 and mammalian target of rapamycin (mTOR) expression in patients with diffuse large B-cell lymphoma. Leuk Lymphoma. 2018;59(6):1451-1460.

10. Reinholdt L, Laursen MB, Schmitz A, et al. The CXCR4 antagonist plerixafor enhances the effect of rituximab in diffuse large B-cell lymphoma cell lines. Biomark Res. 2016;4:12.

11. Domanska UM, Kruizinga RC, Nagengast WB, et al. A review on CXCR4/CXCL12 axis in oncology: No place to hide. Eur J Cancer. 2013;49(1):219-230.

12. Bertolini F, Dell'Agnola C, Mancuso P, et al. CXCR4 neutralization, a novel therapeutic approach for non-Hodgkin's lymphoma. Cancer Res. 2002;62(11):3106-3112.

13. Kuhne MR, Mulvey $\mathrm{T}$, Belanger $\mathrm{B}$, et al BMS-936564/MDX-1338: a fully human anti-CXCR4 antibody induces apoptosis in vitro and shows antitumor activity in vivo in hematologic malignancies. Clin Cancer Res. 2013;19(2):357-366

14. Reinholdt LR, Laursen MB, Falgreen $S$, et al.
High expression of CXCR4 impairs antiCD20 monoclonal antibody (Rituximab)dependent cytotoxicity in diffuse large B-cell lymphoma. Blood. 2015; 126(23):1455-1455.

15. Beider K, Ribakovsky E, Abraham M, et al Targeting the CD20 and CXCR4 pathways in non-hodgkin lymphoma with rituximab and high-affinity CXCR4 antagonist BKT140. Clin Cancer Res. 2013;19(13): 3495-3507.

16. Unzueta U, Céspedes MV, Ferrer-Miralles $\mathrm{N}$, et al. Intracellular CXCR4+ cell targeting with T22-empowered protein-only nanoparticles. Int J Nanomedicine. 2012; 7:4533-4544.

17. Céspedes MV, Unzueta U, Tatkiewicz W, et al. In vivo architectonic stability of fully de novo designed protein-only nanoparticles. ACS Nano. 2014;8(5):4166-4176.

18. Sánchez-García L, Serna N, Álamo P, et al. Self-assembling toxin-based nanoparticles as self-delivered antitumoral drugs. J Control Release. 2018;274:81-92.

19. Serna N, Sánchez-García L, Unzueta U, et al. Protein-Based Therapeutic Killing for Cancer Therapies. Trends Biotechnol. 2018;36(3):318-335

20. Rosenblum D, Joshi N, Tao W, Karp JM Peer D. Progress and challenges towards targeted delivery of cancer therapeutics. Nat Commun. 2018;9(1):1410.

21. Pirollo KF, Chang EH. Does a targeting ligand influence nanoparticle tumor localization or uptake? Trends Biotechnol. 2008; 26(10):552-558 
